\title{
Hepatitis B virus in drug users in France: prevalence and vaccination history, ANRS-Coquelicot Survey 2011-2013
}

\author{
C. BROUARD ${ }^{1}$, J. PILLONEL ${ }^{1}$, P. SOGNI ${ }^{2,3,4}$, A. CHOLLET ${ }^{5}$, J. V. LAZARUS ${ }^{6,7}$, \\ X. PASCAL ${ }^{5}$, F. BARIN ${ }^{8}$, M. JAUFFRET-ROUSTIDE*1,5 and the ANRS Coquelicot \\ Survey Group $\dagger$ \\ ${ }^{1}$ Santé publique France, Direction des Maladies Infectieuses, Saint-Maurice, France \\ ${ }^{2}$ Université Paris Descartes, Paris, France \\ ${ }^{3}$ Inserm U1223, Institut Pasteur, France \\ ${ }^{4}$ Assistance Publique des Hôpitaux de Paris, Hôpital Cochin, Service d'Hépatologie, Paris, France \\ ${ }^{5}$ Cermes3, Inserm U988, UMR CNRS 8211, Université Paris Descartes, EHESS, Paris, France \\ ${ }^{6}$ Centre for Health and Infectious Disease Research and WHO Collaborating Centre on HIV and Viral Hepatitis, \\ Rigshospitalet, University of Copenhagen, Copenhagen, Denmark \\ ${ }^{7}$ ISGlobal, Hospital Clínic, University of Barcelona, Barcelona, Spain \\ ${ }^{8}$ Inserm U966, Centre National de Référence du VIH, CHU Bretonneau, Université François-Rabelais, Tours, \\ France
}

Received 4 October 2016; Final revision 24 November 2016; Accepted 30 November 2016; first published online 19 January 2017

\section{SUMMARY}

People who use drugs (PWUD) are a key population for hepatitis B virus (HBV) vaccination and screening. We aimed to estimate the seroprevalence of HBs antigen (HBsAg) and self-reported HBV vaccination history in French PWUD attending harm reduction centres using data from the ANRS-Coquelicot multicentre survey conducted in 2011-2013 in 1718 PWUD. Self-fingerprick blood samples were collected on dried blood spots to detect the presence of HBsAg. HBsAg seroprevalence was estimated at 1.4\% [95\% confidence interval (CI) $0 \cdot 8-2 \cdot 5]$. It varied between PWUD born in high $(7 \cdot 6 \%, 95 \%$ CI $2 \cdot 7-19 \cdot 1)$, moderate $(2 \cdot 2 \%, 95 \%$ CI $0 \cdot 8-5 \cdot 7)$ and low $(0 \cdot 7 \%$, $95 \%$ CI $0 \cdot 3-1 \cdot 5)$ endemic zones. Factors independently associated with HBsAg carriage were being born in a moderate or high endemic zone or reporting precarious housing. Self-reported HBV vaccination history varied from $47 \cdot 4 \%$ in high endemic zones, to $59 \cdot 3 \%$ and $62 \cdot 6 \%$ for moderate and low endemic zones, respectively. Our results suggest that drug use plays a small and substantial role, respectively, in HBsAg carriage in PWUD born in high/moderate and low endemic zones.

Key words: Estimating, France, hepatitis B, people who use drugs (PWUD), prevalence of disease, vaccination (immunization).

\section{INTRODUCTION}

It is estimated that 248 million people are currently infected with the hepatitis B virus (HBV) worldwide

\footnotetext{
* Author for correspondence: Mrs M. Jauffret-Roustide, Cermes3, 45 rue des Saints-Pères, 75006 Paris, France,

(Email: marie.jauffretroustide@santepubliquefrance.fr; marie.jauffret-roustide@inserm.fr)

$\uparrow$ Members of the ANRS Coquelicot Survey Group are given in the Appendix.
}

[1], of whom 686000 die every year [2]. This virus is transmitted through unprotected sex, direct contact with infected blood and from mother to child during childbirth. Over $95 \%$ of adult infections resolve spontaneously whereas $95 \%$ of neonates and $30-50 \%$ of cases in children aged 1-4 years develop chronic infection. Chronic infection can remain asymptomatic for several years and can lead to several complications, including liver cirrhosis and hepatocellular carcinoma. 
The fight to stop the spread of HBV infection includes prevention, vaccination, and screening. For HBV-infected individuals, antiviral therapy is effective in suppressing HBV replication and reducing the risks of developing complications [3].

The epidemiology of the disease varies greatly according to geographical zone. In high HBsAg prevalence areas $(8 \%$ in both sub-Saharan Africa and Asia), the risk of being infected during one's life is greater than $60 \%$, with most infections being acquired at birth or during the first years of life. Conversely, in low-prevalence endemic countries (HBsAg prevalence $<2 \%$ ), HBV transmission usually occurs in adulthood, mainly via sexual activity, and the disease resolves spontaneously in most cases $[1,3]$.

With a HBsAg prevalence estimated at $0.65 \%$ in the adult general population, mainland France is a low-HBV prevalence country [4]. When the HBV vaccine became available in France in 1980, it was first targeted at high-risk populations, including individuals with multiple sexual partners, people who inject drugs (PWID), healthcare workers and travellers to high or moderate HBV-prevalence countries. In accordance with World Health Organization (WHO) guidelines [5], in 1995, France launched a mass HBV vaccination campaign in schools targeting infants and pre-adolescents. The campaign ended 3 years later because of controversy surrounding the possible relationship, now refuted, between HBV vaccination and demyelination [6].

In 2010, at the WHO 63rd World Health Assembly, a resolution was adopted to establish 'goals and strategies for disease control, increasing education and promoting screening and treatment' of people infected with $\mathrm{HBV}$ and hepatitis $\mathrm{C}$ virus (HCV) [7]. In this resolution, people who use drugs (PWUD) were considered a key population for viral hepatitis vaccination and screening. This is because they are at high risk of contracting HBV through the sharing of needles and because of frequent precarious life conditions [8]. To assess the impact of the implementation of the WHO resolution on both HBsAg prevalence and HBV vaccine coverage associated data are needed. In 2011-2013, the ANRS-Coquelicot survey was conducted in PWUD in France with the main objective of estimating HCV and HIV prevalence [9]. This paper presents estimates of HBsAg seroprevalence and self-reported HBV vaccination history in PWUD using data from the ANRS-Coquelicot survey.

\section{METHODS}

\section{Study design}

The methods used have been described elsewhere $[9,10]$. Briefly, ANRS-Coquelicot is a French cross-sectional epidemiological survey of PWUD recruited in harm reduction programmes and care centres in five cities (Lille, Strasbourg, Paris, Bordeaux, Marseille) and two departments (administrative districts) beside Paris (Seine-Saint-Denis and Seine-et-Marne) in 2011-2013. In each location, an exhaustive inventory of all structures providing services to drug users was performed and the number of PWUD eligible for the study was evaluated. Participants were recruited using a two-stage sampling plan, first by random selection of the centres and then by random selection of individuals attending these centres. Inclusion criteria were as follows: aged $\geqslant 18$ years, having injected or snorted during lifetime, and able to speak French or Russian (Russian speakers represented $\sim 30 \%$ of those eligible for inclusion in some services).

\section{Data collection}

Data was collected using a socio-behavioural questionnaire administered through face-to-face interviews. Interviewers were independent of all the centres. The questionnaire explored: (1) sociodemographic characteristics (age, gender, country of birth, level of education, employment, household and living conditions); (2) health status (self-perceived health, history of HBV vaccination); (3) drug use (drugs consumed and modes of consumption); (4) drug-related at-risk practices during lifetime and during the previous month (sharing syringes and other injection paraphernalia); (5) sexual risk-taking practices during the previous 12 months.

Self-fingerprick blood sampling on dried blood spots (DBS) was performed.

Data collection was anonymous and informed consent to participate in the study was obtained orally from the person being interviewed. The research protocol was validated by the French ethical committee on biomedical research (no. 2010-A01330-39).

\section{Laboratory method}

Testing for HBsAg, anti-HCV and anti-HIV antibodies $(\mathrm{Ab})$ was performed on blood samples collected on DBS. The relevance of DBS for HBsAg screening has been repeatedly shown $[11,12]$. Preliminary validation studies showed that the 
sensitivity of HBsAg detection in samples collected on DBS is about 100 times lower than in standard procedures on serum or plasma (ANRS-Prevagay study 2009, unpublished data). However, this lower sensitivity did not hamper the detection of HBV infection due to the very large amount of HBsAg circulating in the blood of most of the infected individuals studied: between $10^{2} \mathrm{IU} / \mathrm{ml}$ and $10^{4} \mathrm{IU} / \mathrm{ml}$ in HBeAg-negative and $\mathrm{HBeAg}$-positive subjects, respectively, the sensitivity of the Monolisa HBsAg Ultra reagent being 0.05 $\mathrm{IU} / \mathrm{ml}$ [13]. As the probability of patients developing acute HBV during our study was very low, the presence of HBsAg in the blood was considered a sign of chronic infection. ELISA kits were used for detection of anti-HIV Ab (Genscreen HIV Ac/Ac Bio-Rad, USA) and anti-HCV Ab (anti-HCV Ortho, USA) [9, 10].

\section{Statistical analysis}

Data analyses took into account the time-location sampling design [10]. To take into account the possibility that PWUD attended more than one participating service during data collection, and to obtain bias-free estimators, the Generalized Weight Share Measure (GWSM) was used [10].

Self-reported HBV vaccination history was estimated from answers to the question, 'Have you ever been vaccinated against hepatitis B?' irrespective of the number of doses. Univariate analyses of estimates of HBsAg prevalence and self-reported HBV vaccination history were performed according to socio-demographic characteristics, HIV and HCV co-infection status, drug consumption and risk-taking practices. Analyses were stratified according to HBV prevalence ('high', 'moderate', 'low') in PWUD country of birth [7]. Sub-Saharan Africa and Eastern Asia were classified as high endemic zones, Northern Africa, the Middle East and Central and Eastern Europe as moderate endemic zones and Western Europe and North America as low endemic zones.

The age variable was dichotomized to compare PWUD aged $<35$ years at the time of the study with those aged $\geqslant 35$ years. This value was chosen as the former were more likely to have benefited from harm reduction measures, while the latter's practices and exposure to HBV infection evolved simultaneously with the implementation of harm reduction tools [10]. Respondents who declared having had sexual relationships with multiple partners and those who had a sexual relationship with only one person but did not consistently use condoms, were considered to have taken sexual risks in the previous 12 months.

Two multivariate Poisson regression models were then used to identify factors independently associated with HBsAg seroprevalence and HBV vaccine coverage, respectively. In order to evaluate the role of endemicity level in the country of birth, these models were not stratified on this variable.

Analyses were performed with Stata v. 12 (StataCorp. USA).

\section{RESULTS}

All the 138 centres solicited participated in the survey. PWUD participation rate was $75 \%(n=1718)$, with $92 \%$ of the latter agreeing to provide a fingerprick blood sample. A total of 1575 samples were analysed. No difference was observed in terms of age and gender between respondents and non-respondents, or between those who agreed to provide a blood sample and those who refused.

Respondents were mainly men $(79 \cdot 6 \%)$ aged $\geqslant 35$ years $(69 \cdot 1 \%)$. More than one in six PWUD reported precarious housing (i.e. sleeping in a squat or on the streets). Nearly two thirds of PWUD declared a history of injection during their lifetime and $37.0 \%$ during the previous month. Other PWUD characteristics are described in Table 1.

Overall, HBsAg seroprevalence was $1.4 \%[95 \%$ confidence interval (CI) $0 \cdot 8-2 \cdot 5$ ]. Seroprevalence in PWUD from high, moderate and low endemic zones was $7 \cdot 6 \%(95 \%$ CI $2 \cdot 7-19 \cdot 1), 2 \cdot 2 \%(95 \%$ CI $0 \cdot 8-5 \cdot 7)$ and $0 \cdot 7 \%(95 \%$ CI $0 \cdot 3-1 \cdot 5)$, respectively (Table 2$)$.

With respect to HBV vaccination, $11.9 \%$ of PWUD declared that they did not know whether they had been vaccinated $(n=188)$. After exclusion of these and seven non-respondents, self-reported HBV vaccination history was $60 \cdot 9 \%(95 \%$ CI 56.2-65.4). It varied according to the endemicity level in the country of birth: from $47 \cdot 4 \%$ to $59 \cdot 3 \%$ to $62 \cdot 6 \%$ for those born in high, moderate and low endemic zones, respectively (Table 2).

HBsAg seroprevalence in PWUD who declared they were vaccinated against $\mathrm{HBV}$, and in those who declared they were not vaccinated was $0 \cdot 8 \%$ (95\% CI $0 \cdot 3-1 \cdot 8)$ and $2 \cdot 5 \%(95 \%$ CI $1 \cdot 0-5 \cdot 9)$, respectively.

\section{HBsAg prevalence and self-reported $\mathrm{HBV}$ vaccination history in PWUD born in low endemic zones}

For PWUD born in low endemic zones, HBsAg prevalence was higher in those who were not in a 
Table 1. Socio-demographic characteristics, health status and both sexual and drug-use at-risk practices according to the endemicity level of PWUD country of birth, ANRS-Coquelicot Survey 2011-2013

\begin{tabular}{|c|c|c|c|}
\hline & $\begin{array}{l}\text { Low endemic zone } \\
(n=1233) \\
(95 \% \mathrm{CI})\end{array}$ & $\begin{array}{l}\text { Moderate/high endemic } \\
\text { zone }(n=485) \\
(95 \% \mathrm{CI})\end{array}$ & $\begin{array}{l}\text { Total } \\
(n=1718) \\
(95 \% \mathrm{CI})\end{array}$ \\
\hline \multicolumn{4}{|l|}{ Gender } \\
\hline Male & $74 \cdot 3(69 \cdot 0-79 \cdot 0)$ & $95 \cdot 8(92 \cdot 8-97 \cdot 6)$ & $79 \cdot 6(75 \cdot 3-83 \cdot 3)$ \\
\hline Female & $25 \cdot 7(21 \cdot 0-31 \cdot 0)$ & $4 \cdot 2(2 \cdot 4-7 \cdot 2)$ & $20 \cdot 4(16 \cdot 7-24 \cdot 7)$ \\
\hline \multicolumn{4}{|l|}{ Age } \\
\hline$<35$ years & $33 \cdot 4(29 \cdot 0-38 \cdot 1)$ & $23 \cdot 2(17 \cdot 2-30 \cdot 4)$ & $30 \cdot 9(27 \cdot 2-34 \cdot 8)$ \\
\hline$\geqslant 35$ years & $66 \cdot 6(61 \cdot 9-71 \cdot 0)$ & $76 \cdot 8(69 \cdot 6-82 \cdot 8)$ & $69 \cdot 1(65 \cdot 2-72 \cdot 8)$ \\
\hline \multicolumn{4}{|l|}{ Russian speaking } \\
\hline Yes & 0 & $7 \cdot 0(4 \cdot 2-11 \cdot 6)$ & $1 \cdot 7(1 \cdot 0-2 \cdot 9)$ \\
\hline \multicolumn{4}{|l|}{ In a relationship } \\
\hline Yes & $49 \cdot 4(45 \cdot 0-53 \cdot 9)$ & $36 \cdot 5(29 \cdot 7-43 \cdot 9)$ & $46 \cdot 3(42 \cdot 8-49 \cdot 7)$ \\
\hline \multicolumn{4}{|l|}{ Educational level } \\
\hline >High school certificate & $25 \cdot 0(19 \cdot 3-31 \cdot 8)$ & $21 \cdot 8(16 \cdot 3-28 \cdot 5)$ & $24 \cdot 2(19 \cdot 9-29 \cdot 2)$ \\
\hline$<$ High school certificate & $72 \cdot 1(65 \cdot 9-77 \cdot 5)$ & $61 \cdot 5(53 \cdot 9-68 \cdot 5)$ & $69 \cdot 5(63 \cdot 9-74 \cdot 5)$ \\
\hline Primary school & $2 \cdot 9(1 \cdot 8-4 \cdot 7)$ & $16 \cdot 7(11 \cdot 0-24 \cdot 6)$ & $6 \cdot 3(4 \cdot 9-8 \cdot 2)$ \\
\hline \multicolumn{4}{|l|}{ Type of housing } \\
\hline Stable & $84 \cdot 6(77 \cdot 8-89 \cdot 6)$ & $74 \cdot 1(65 \cdot 4-81 \cdot 3)$ & $82 \cdot 1(75 \cdot 3-87 \cdot 3)$ \\
\hline Precarious & $15 \cdot 4(10 \cdot 4-22 \cdot 2)$ & $25 \cdot 9(18 \cdot 7-34 \cdot 6)$ & $17 \cdot 9(12 \cdot 7-24 \cdot 7)$ \\
\hline \multicolumn{4}{|c|}{ Past history of injection (lifetime) } \\
\hline \multirow{2}{*}{\multicolumn{4}{|c|}{ Injection in the previous month }} \\
\hline & & & \\
\hline Yes & $36 \cdot 3(26 \cdot 4-47 \cdot 5)$ & $40 \cdot 0(27 \cdot 3-54 \cdot 2)$ & $37 \cdot 0(27 \cdot 3-47 \cdot 9)$ \\
\hline \multicolumn{4}{|c|}{ Past history of intranasal drug use (lifetime) } \\
\hline Yes & $96 \cdot 9(95 \cdot 1-98 \cdot 1)$ & $96 \cdot 2(93 \cdot 5-97 \cdot 8)$ & $96 \cdot 7(95 \cdot 2-97 \cdot 8)$ \\
\hline \multicolumn{4}{|c|}{ Crack-cocaine consumption (lifetime) } \\
\hline Yes & $68 \cdot 3(61 \cdot 6-74 \cdot 3)$ & $72 \cdot 8(62 \cdot 7-81 \cdot 0)$ & $69 \cdot 4(62 \cdot 7-75 \cdot 4)$ \\
\hline \multicolumn{4}{|c|}{ Smoking crack cocaine (previous month) } \\
\hline Yes & $30 \cdot 0(21 \cdot 2-40 \cdot 6)$ & $40 \cdot 1(28 \cdot 0-53 \cdot 6)$ & $32 \cdot 5(23 \cdot 2-43 \cdot 4)$ \\
\hline \multicolumn{4}{|l|}{ Self-perceived state of health } \\
\hline Poor & $29 \cdot 3(25 \cdot 5-33 \cdot 4)$ & $37 \cdot 9(31 \cdot 6-44 \cdot 6)$ & $31 \cdot 4(28 \cdot 1-34 \cdot 9)$ \\
\hline Good & $70 \cdot 7(66 \cdot 6-74 \cdot 5)$ & $62 \cdot 1(55 \cdot 4-68 \cdot 4)$ & $68 \cdot 6(65 \cdot 1-71 \cdot 9)$ \\
\hline \multicolumn{4}{|l|}{ HIV status } \\
\hline HIV Ab negative & $89 \cdot 1(85 \cdot 7-91 \cdot 7)$ & $94 \cdot 1(90 \cdot 8-96 \cdot 3)$ & $90 \cdot 3(87 \cdot 8-92 \cdot 3)$ \\
\hline HIV Ab positive & $10 \cdot 9(8 \cdot 3-14 \cdot 3)$ & $5 \cdot 9(3 \cdot 7-9 \cdot 2)$ & $9 \cdot 7(7 \cdot 7-12 \cdot 2)$ \\
\hline \multicolumn{4}{|l|}{$\mathrm{HCV}$ status } \\
\hline $\mathrm{HCV}$ Ab negative & $53 \cdot 3(49 \cdot 0-57 \cdot 7)$ & $61 \cdot 2(52 \cdot 5-69 \cdot 3)$ & $55 \cdot 2(50 \cdot 9-59 \cdot 5)$ \\
\hline $\mathrm{HCV} \mathrm{Ab}$ positive & $46 \cdot 7(42 \cdot 3-51 \cdot 0)$ & $38 \cdot 8(30 \cdot 7-47 \cdot 5)$ & $44 \cdot 8(40 \cdot 5-49 \cdot 1)$ \\
\hline \multicolumn{4}{|l|}{ Syringe sharing (lifetime) } \\
\hline Yes & $61 \cdot 6(56 \cdot 6-66 \cdot 4)$ & $64 \cdot 9(56 \cdot 4-72 \cdot 6)$ & $62 \cdot 2(57 \cdot 6-66 \cdot 6)$ \\
\hline \multicolumn{4}{|c|}{ Sexual risk taking practices (previous 12 months) } \\
\hline Yes & $64 \cdot 2(60 \cdot 3-68 \cdot 0)$ & $58 \cdot 5(51 \cdot 1-65 \cdot 6)$ & $62 \cdot 8(59 \cdot 4-66 \cdot 1)$ \\
\hline
\end{tabular}

PWUD, People who use drugs; CI, confidence interval.

relationship $\left(1 \cdot 3 \%\right.$ vs. $\left.0 \cdot 1 \%, P<10^{-3}\right)$, in those with only a primary (or lower) level of education $(8 \cdot 8 \%)$ and in those who were living in precarious housing conditions $\left(2 \cdot 9 \%\right.$ vs. $\left.0 \cdot 3 \%, P<10^{-2}\right)$ (Table 3$)$. It was also higher in PWUD who had used crack cocaine in the previous month $(1.7 \%$ vs. $0.2 \%, P<0.05)$ and those who had already shared syringes during their lifetime $\left(1 \cdot 1 \%\right.$ vs. $\left.0 \%, P<10^{-3}\right)$.
Self-reported HBV vaccination history was more significantly frequent in PWUD aged $<35$ years $\left(73 \cdot 1 \%\right.$ vs. $\left.57 \cdot 8 \%, P<10^{-3}\right)$ and in those who were in a relationship $\left(67 \cdot 3 \%\right.$ vs. $\left.57 \cdot 6 \%, P<10^{-2}\right)$. It increased with level of education, from $34 \cdot 1 \%$ for those with only primary school education to $67.9 \%$ for those who had third-level education $(P<0 \cdot 05)$. HBV vaccination history was also more frequent in PWUD with 
Table 2. HBs Ag seroprevalence and self-reported HBV vaccination history according to the endemicity level of PWUD country of birth, ANRS-Coquelicot Survey 2011-2013

\begin{tabular}{|c|c|c|}
\hline & $\begin{array}{l}\text { HBs Ag seroprevalence } \\
(95 \% \mathrm{CI})\end{array}$ & $\begin{array}{l}\text { Self-reported HBV } \\
\text { vaccination history } \\
(95 \% \mathrm{CI})\end{array}$ \\
\hline $\begin{array}{l}\text { Low endemic zone } \\
\qquad(n=1125)\end{array}$ & $\begin{array}{l}0 \cdot 7 * * *(0 \cdot 3-1 \cdot 5) \\
(n=7)\end{array}$ & $\begin{array}{l}62 \cdot 6(57 \cdot 6-67 \cdot 4) \\
(n=686)\end{array}$ \\
\hline $\begin{array}{l}\text { Moderate endemic zone } \\
\quad(n=348)\end{array}$ & $\begin{array}{l}2 \cdot 2 * * *(0 \cdot 8-5 \cdot 7) \\
(n=7)\end{array}$ & $\begin{array}{l}59 \cdot 3(48 \cdot 8-68 \cdot 9) \\
(n=179)\end{array}$ \\
\hline $\begin{array}{l}\text { High endemic zone } \\
\qquad(n=102)\end{array}$ & $\begin{array}{l}7 \cdot 6 * * *(2 \cdot 7-19 \cdot 1) \\
(n=9)\end{array}$ & $\begin{array}{l}47 \cdot 4(29 \cdot 9-65 \cdot 5) \\
(n=60)\end{array}$ \\
\hline $\begin{array}{l}\text { Total } \\
\qquad(n=1575)\end{array}$ & $\begin{array}{l}1 \cdot 4(0 \cdot 8-2 \cdot 5) \\
(n=23)\end{array}$ & $\begin{array}{l}60 \cdot 9(56 \cdot 2-65 \cdot 4) \\
(n=925)\end{array}$ \\
\hline
\end{tabular}

PWUD, People who use drugs; CI, confidence interval.

$\dagger$ Vaccination history was estimated after exclusion of PWUD who were unaware of their vaccination status $(n=188)$ and non-respondents $(n=7)$.

*** $P<0 \cdot 01$.

HCV seronegative status $(69 \cdot 3 \%)$ than in PWUD with seropositive HCV status (55.8\%), and in those with HIV seronegative status $(64 \cdot 4 \%)$ than in those with HIV seropositive status $(49 \cdot 4 \%)$. It was also more frequent in PWUD who had a positive perception of their health status $(66.1 \%)$ than in those who had a negative perception $(53.9 \%, P<0.05)$. PWUD who had already engaged in sexual risk-taking behaviours declared they were vaccinated more often than those who had not engaged in such practices $(66.0 \%$ vs. $56.5 \%, P<0.05)$.

\section{HBsAg prevalence and self-reported $\mathrm{HBV}$ vaccination history in PWUD born in moderate/high endemic zones}

$\mathrm{HBs} A g$ prevalence was significantly higher in $\mathrm{HCV}$ seronegative PWUD $\left(6 \cdot 2 \%\right.$ vs. $\left.0 \cdot 2 \%, P<10^{-3}\right)$ and in those who had never injected $(6.0 \%$ vs. $1.4 \%, P<0.05)$. It was 12 times higher in PWUD who did not report any history of HBV vaccination $(7 \cdot 4 \%)$ than in those who declared they were vaccinated $(0 \cdot 6 \%, P<0 \cdot 05)$ (Table 3 ).

Self-reported HBV vaccination history was least frequent in PWUD who had only primary school education $(41 \cdot 1 \%)$.

\section{Multivariate analysis}

HBsAg prevalence was significantly higher in PWUD born in moderate or high endemic zones [prevalence ratio (PR) 4.8] and in those who reported precarious housing (PR 3.6) (Table 4).

Self-reported vaccination history was less frequent in PWUD speaking Russian (PR 0.85), being aged $\geqslant 35$ years (PR 0.88 ) or having only primary school education (PR 0.63). Conversely, self-reported vaccination history was significantly more frequent in PWUD with a positive perception of their health (PR 1·19) (Table 5).

\section{DISCUSSION}

The ANRS-Coquelicot survey is the first epidemiological study in France to provide national estimates of HBV chronic infection prevalence based on biological data and self-reported HBV vaccination history from a large sample of PWUD. This survey was innovative in that it coupled time-location sampling with GWSM. Furthermore, respondents were recruited from an exhaustive number of harm reduction facilities and care centres. The response rates to participate in the survey and to undergo fingerprick blood testing were very high.

HBsAg seroprevalence estimated in our study $(1 \cdot 4 \%, 95 \%$ CI $0 \cdot 8-2 \cdot 5)$ was similar to that estimated in 2004 for the subgroup of the French adult general population which declared previous drug injection $(1 \cdot 9 \%, 95 \%$ CI $0 \cdot 2-15 \cdot 6)$ [4]. Our HBsAg prevalence estimate ranked France among those European countries where HBV is least endemic in PWUD: $1.5 \%$ for 2011 in Germany [14], 2.0\% for 2012 in Italy [15] and $0.57 \%$ for 2013 in the UK [16].

In our study, as expected, the proportion of PWUD infected with HBV reflected the endemicity level in their country of birth, from $0.7 \%$ (95\% CI $0 \cdot 3-1 \cdot 5)$ for PWUD born in low endemic zones to 7.6\% (95\% CI 2.7-19.1) for those born in high endemic zones. 
Table 3. HBs Ag prevalence and self-reported HBV vaccination history according to PWUD socio-demographic characteristics, health status and both sexual and drug-use at-risk practices, ANRS-Coquelicot Survey 2011-2013

\begin{tabular}{|c|c|c|c|c|c|c|}
\hline & \multicolumn{2}{|c|}{ Low endemic zone $(n=1125)$} & \multicolumn{2}{|c|}{ Moderate/high endemic zone $(n=450)$} & \multicolumn{2}{|l|}{ Total $(n=1575)$} \\
\hline & $\begin{array}{l}\mathrm{HBs} \mathrm{Ag} \\
\text { seroprevalence } \\
(95 \% \mathrm{CI})\end{array}$ & $\begin{array}{l}\text { Self-reported HBV } \\
\text { vaccination history } \dagger \\
(95 \% \mathrm{CI})\end{array}$ & $\begin{array}{l}\mathrm{HBs} \mathrm{Ag} \\
\text { seroprevalence } \\
(95 \% \mathrm{CI})\end{array}$ & $\begin{array}{l}\text { Self-reported HBV } \\
\text { vaccination history } \dagger \\
(95 \% \mathrm{CI})\end{array}$ & $\begin{array}{l}\mathrm{HBs} \mathrm{Ag} \\
\text { seroprevalence } \\
(95 \% \mathrm{CI})\end{array}$ & $\begin{array}{l}\text { Self-reported HBV } \\
\text { vaccination history } \dagger \\
(95 \% \mathrm{CI})\end{array}$ \\
\hline \multicolumn{7}{|l|}{ Gender } \\
\hline Male & $0 \cdot 7(0 \cdot 3-1 \cdot 7)$ & $62 \cdot 1(56 \cdot 5-67 \cdot 5)$ & $3 \cdot 8(1 \cdot 7-8 \cdot 4)$ & $55 \cdot 8(44 \cdot 3-66 \cdot 7)$ & $1 \cdot 6(0 \cdot 9-2 \cdot 9)$ & $60 \cdot 3(55 \cdot 3-65 \cdot 0)$ \\
\hline Female & $0 \cdot 5(0 \cdot 1-3 \cdot 6)$ & $63 \cdot 7(55 \cdot 2-71 \cdot 3)$ & $3 \cdot 6(0 \cdot 6-19 \cdot 3)$ & $49 \cdot 9(31 \cdot 9-67 \cdot 9)$ & $0 \cdot 7(0 \cdot 1-3 \cdot 1)$ & $62 \cdot 9(54 \cdot 9-70 \cdot 2)$ \\
\hline \multicolumn{7}{|l|}{ Age } \\
\hline$<35$ years & $0 \cdot 0$ & $73 \cdot 1 * * *(65 \cdot 2-79 \cdot 8)$ & $6 \cdot 8(2 \cdot 2-19 \cdot 3)$ & $48 \cdot 2(30 \cdot 2-66 \cdot 6)$ & $1 \cdot 3(0 \cdot 4-4 \cdot 0)$ & $68 \cdot 5 *(60 \cdot 7-75 \cdot 4)$ \\
\hline$\geqslant 35$ years & $1 \cdot 0(0 \cdot 4-2 \cdot 2)$ & $57 \cdot 8^{* * *}(52 \cdot 1-63 \cdot 2)$ & $2 \cdot 8(1 \cdot 0-7 \cdot 9)$ & $57 \cdot 8(44 \cdot 1-70 \cdot 4)$ & $1 \cdot 5(0 \cdot 8-2 \cdot 8)$ & $57 \cdot 8 *(52 \cdot 2-63 \cdot 1)$ \\
\hline \multicolumn{7}{|l|}{ Russian speaking } \\
\hline No & n.c. & n.c. & $4 \cdot 0(1 \cdot 7-8 \cdot 8)$ & $56 \cdot 4(44 \cdot 6-67 \cdot 5)$ & $1 \cdot 4(0 \cdot 8-2 \cdot 5)$ & $61 \cdot 2 * *(56 \cdot 4-65 \cdot 7)$ \\
\hline Yes & n.c. & n.c. & $1 \cdot 1(0 \cdot 3-4 \cdot 5)$ & $45 \cdot 6(33 \cdot 8-57 \cdot 8)$ & $1 \cdot 1(0 \cdot 3-4 \cdot 5)$ & $45 \cdot 5 * *(33 \cdot 8-57 \cdot 8)$ \\
\hline \multicolumn{7}{|l|}{ In a relationship } \\
\hline No & $1 \cdot 3 * * *(0 \cdot 5-3 \cdot 0)$ & $57 \cdot 6^{* *}(52 \cdot 1-62 \cdot 9)$ & $3 \cdot 8(1 \cdot 6-8 \cdot 6)$ & $56 \cdot 7(44 \cdot 3-68 \cdot 3)$ & $2 \cdot 0(1 \cdot 2-3 \cdot 5)$ & $57 \cdot 3 *(51 \cdot 6-62 \cdot 9)$ \\
\hline Yes & $0 \cdot 1 * * *(0-0 \cdot 5)$ & $67 \cdot 3 * *(60 \cdot 8-73 \cdot 2)$ & $3 \cdot 6(1 \cdot 1-11 \cdot 4)$ & $54 \cdot 0(38 \cdot 9-68 \cdot 5)$ & $0 \cdot 8(0 \cdot 2-2 \cdot 4)$ & $64 \cdot 7 *(58 \cdot 8-70 \cdot 2)$ \\
\hline \multicolumn{7}{|l|}{ Educational level } \\
\hline >High school certificate & $0 \cdot 3 * * *(0-2 \cdot 5)$ & $67 \cdot 9 *(57 \cdot 9-76 \cdot 5)$ & $2 \cdot 8(0 \cdot 5-14 \cdot 8)$ & $42 \cdot 4 * *(26 \cdot 8-59 \cdot 7)$ & $0 \cdot 8(0 \cdot 1-4 \cdot 8)$ & $62 \cdot 4 *(53 \cdot 9-70 \cdot 2)$ \\
\hline$<$ High school certificate & $0 \cdot 5^{* * *}(0 \cdot 2-1 \cdot 5)$ & $62 \cdot 0 *(56 \cdot 4-67 \cdot 3)$ & $4 \cdot 0(1 \cdot 6-10 \cdot 0)$ & $64 \cdot 2 * *(52 \cdot 6-74 \cdot 4)$ & $1 \cdot 3(0 \cdot 6-2 \cdot 6)$ & $62 \cdot 5 *(57 \cdot 2-67 \cdot 4)$ \\
\hline Primary school & $8 \cdot 8 * * *(1 \cdot 2-43 \cdot 1)$ & $34 \cdot 1 *(14 \cdot 5-61 \cdot 2)$ & $4 \cdot 1(0 \cdot 6-22 \cdot 0)$ & $41 \cdot 1 * *(24 \cdot 0-60 \cdot 5)$ & $5 \cdot 6(1 \cdot 5-19 \cdot 0)$ & $38 \cdot 5 *(58 \cdot 8-70 \cdot 2)$ \\
\hline \multicolumn{7}{|l|}{ Type of housing } \\
\hline Stable & $0 \cdot 3 * *(0 \cdot 1-1 \cdot 1)$ & $62 \cdot 9(57 \cdot 6-68 \cdot 0)$ & $3 \cdot 0(1 \cdot 2-7 \cdot 2)$ & $53 \cdot 9(41 \cdot 3-66 \cdot 1)$ & $0 \cdot 9 * *(0 \cdot 4-2 \cdot 1)$ & $60 \cdot 9(56 \cdot 3-65 \cdot 4)$ \\
\hline Precarious & $2 \cdot 9 * *(1 \cdot 0-8 \cdot 4)$ & $60 \cdot 8(51 \cdot 1-69 \cdot 7)$ & $6 \cdot 2(1 \cdot 6-21 \cdot 2)$ & $60 \cdot 5(43 \cdot 4-75 \cdot 3)$ & $4 \cdot 0 * *(1 \cdot 8-8 \cdot 9)$ & $60 \cdot 7(50 \cdot 2-70 \cdot 3)$ \\
\hline \multicolumn{7}{|c|}{ Past history of injection (lifetime) } \\
\hline No & $0 \cdot 6(0 \cdot 1-3 \cdot 3)$ & $68(58 \cdot 8-75 \cdot 9)$ & $6 \cdot 0 *(2 \cdot 4-14 \cdot 2)$ & $51 \cdot 6(36 \cdot 3-66 \cdot 6)$ & $2 \cdot 7 *(1 \cdot 2-5 \cdot 7)$ & $61 \cdot 7(54 \cdot 6-68 \cdot 3)$ \\
\hline Yes & $0 \cdot 7(0 \cdot 2-1 \cdot 9)$ & $60 \cdot 6(55 \cdot 1-65 \cdot 8)$ & $1 \cdot 4 *(0 \cdot 4-4 \cdot 6)$ & $60(50 \cdot 5-68 \cdot 7)$ & $0 \cdot 8 *(0 \cdot 4-1 \cdot 8)$ & $60 \cdot 5(55 \cdot 6-65 \cdot 2)$ \\
\hline \multicolumn{7}{|c|}{ Injection in the previous month } \\
\hline No & $0 \cdot 5(0 \cdot 1-2 \cdot 0)$ & $59 \cdot 3(52 \cdot 6-65 \cdot 6)$ & $2 \cdot 2 * * *(0 \cdot 6-7 \cdot 7)$ & $66 \cdot 4(55 \cdot 6-75 \cdot 8)$ & $0 \cdot 8(0 \cdot 3-2 \cdot 0)$ & $60 \cdot 5(54 \cdot 8-66 \cdot 0)$ \\
\hline Yes & $1 \cdot 0(24 \cdot 1-28 \cdot 5)$ & $62 \cdot 8(52 \cdot 4-72 \cdot 2)$ & $0 \cdot 1 * * *(0 \cdot 0-0 \cdot 5)$ & $50 \cdot 2(32 \cdot 0-68 \cdot 4)$ & $0 \cdot 9(0 \cdot 2-3 \cdot 3)$ & $60 \cdot 4(50 \cdot 3-69 \cdot 6)$ \\
\hline \multicolumn{7}{|c|}{ Past history of intranasal drug use (lifetime) } \\
\hline No & $0 \cdot 0$ & $46 \cdot 0(27 \cdot 0-66 \cdot 3)$ & $4 \cdot 0(0 \cdot 7-18 \cdot 8)$ & $45 \cdot 7(29 \cdot 2-63 \cdot 2)$ & $1 \cdot 1(0 \cdot 2-6 \cdot 7)$ & $45 \cdot 9(31 \cdot 1-61 \cdot 5)$ \\
\hline Yes & $0 \cdot 7(0 \cdot 3-1 \cdot 5)$ & $63 \cdot 2(58 \cdot 0-68 \cdot 2)$ & $3 \cdot 7(1 \cdot 7-8 \cdot 1)$ & $56 \cdot 1(44 \cdot 6-67 \cdot 0)$ & $1 \cdot 4(0 \cdot 8-2 \cdot 5)$ & $61 \cdot 5(56 \cdot 5-66 \cdot 2)$ \\
\hline \multicolumn{7}{|c|}{ Crack-cocaine consumption (lifetime) } \\
\hline No & $0 \cdot 0 * * *(0-0 \cdot 2)$ & $61 \cdot 2(54 \cdot 0-67 \cdot 9)$ & $5 \cdot 8(2 \cdot 2-14 \cdot 4)$ & $46 \cdot 2(32 \cdot 7-60 \cdot 2)$ & $1 \cdot 3(0 \cdot 5-3 \cdot 4)$ & $58 \cdot 0(51 \cdot 9-63 \cdot 9)$ \\
\hline Yes & $0 \cdot 9 * * *(0 \cdot 4-2 \cdot 1)$ & $63 \cdot 3(57 \cdot 1-69 \cdot 1)$ & $3 \cdot 0(1 \cdot 1-7 \cdot 8)$ & $58 \cdot 9(45 \cdot 7-70 \cdot 9)$ & $1 \cdot 5(0 \cdot 8-2 \cdot 6)$ & $62 \cdot 1(56 \cdot 2-67 \cdot 7)$ \\
\hline
\end{tabular}


Smoking crack cocaine (previous month)

\begin{tabular}{|c|c|c|c|c|c|c|}
\hline No & $0 \cdot 2 *(0 \cdot 0-1 \cdot 3)$ & $59 \cdot 6(54 \cdot 0-64 \cdot 9)$ & $3 \cdot 4(1 \cdot 4-7 \cdot 7)$ & $50 \cdot 2(36 \cdot 8-63 \cdot 5)$ & $0.9 *(0 \cdot 4-1 \cdot 9)$ & $57 \cdot 5 *(52 \cdot 9-62 \cdot 0)$ \\
\hline Yes & $1 \cdot 7 *(0 \cdot 7-3 \cdot 8)$ & $69 \cdot 1(59 \cdot 8-77 \cdot 0)$ & $4 \cdot 4(1 \cdot 4-13 \cdot 2)$ & $63 \cdot 4(50 \cdot 5-74 \cdot 7)$ & $2 \cdot 5^{*}(1 \cdot 2-4 \cdot 9)$ & $67 \cdot 4 *(59 \cdot 1-74 \cdot 7)$ \\
\hline \multicolumn{7}{|c|}{ Self-perceived state of health } \\
\hline Poor & $1 \cdot 4(0 \cdot 5-3 \cdot 8)$ & $53 \cdot 9 *(44 \cdot 4-63 \cdot 1)$ & $5 \cdot 5(2 \cdot 0-14 \cdot 3)$ & $47 \cdot 7(32 \cdot 2-63 \cdot 5)$ & $2 \cdot 7 *(1 \cdot 3-5 \cdot 4)$ & $52 \cdot 0 * *(43 \cdot 7-60 \cdot 3)$ \\
\hline Good & $0 \cdot 4(0 \cdot 1-2 \cdot 1)$ & $66 \cdot 1 *(60 \cdot 9-70 \cdot 9)$ & $2 \cdot 7(1 \cdot 0-6 \cdot 7)$ & $60 \cdot 3(49 \cdot 2-70 \cdot 4)$ & $0 \cdot 9 *(0 \cdot 4-1 \cdot 9)$ & $64 \cdot 8 * *(60 \cdot 2-69 \cdot 1)$ \\
\hline \multicolumn{7}{|l|}{ HIV status } \\
\hline HIV Ab negative & $0 \cdot 7(0 \cdot 3-1 \cdot 7)$ & $64 \cdot 4 *(59 \cdot 3-69 \cdot 2)$ & $3 \cdot 6(1 \cdot 5-8 \cdot 4)$ & $59 \cdot 3(48 \cdot 2-69 \cdot 5)$ & $1 \cdot 5(0 \cdot 8-2 \cdot 7)$ & $63 \cdot 1 *(58 \cdot 4-67 \cdot 5)$ \\
\hline HIV Ab positive & $0 \cdot 0$ & $49 \cdot 4 *(34 \cdot 6-64 \cdot 4)$ & $7 \cdot 7(2 \cdot 0-25 \cdot 4)$ & $37 \cdot 9(19 \cdot 9-59 \cdot 9)$ & $1 \cdot 1(0 \cdot 3-4 \cdot 9)$ & $47 \cdot 9 *(35 \cdot 2-60 \cdot 8)$ \\
\hline \multicolumn{7}{|l|}{ HCV status } \\
\hline $\mathrm{HCV} \mathrm{Ab}$ negative & $0 \cdot 3(0 \cdot 1-1 \cdot 7)$ & $69 \cdot 3 *(60 \cdot 8-76 \cdot 7)$ & $6 \cdot 2 * * *(2 \cdot 8-13 \cdot 3)$ & $55 \cdot 9(41 \cdot 1-69 \cdot 7)$ & $1 \cdot 9(0 \cdot 9-3 \cdot 8)$ & $65 \cdot 7 *(60 \cdot 0-71 \cdot 0)$ \\
\hline HCV Ab positive & $1 \cdot 1(0 \cdot 4-3 \cdot 1)$ & $55 \cdot 8 *(48 \cdot 8-62 \cdot 7)$ & $0 \cdot 2 * * *(0 \cdot 1-0 \cdot 8)$ & $61 \cdot 2(51 \cdot 3-70 \cdot 2)$ & $0 \cdot 9(0 \cdot 3-2 \cdot 4)$ & $57 \cdot 0 *(50 \cdot 5-63 \cdot 2)$ \\
\hline \multicolumn{7}{|c|}{ Syringe sharing (lifetime) } \\
\hline No & $0 \cdot 0 * * *(0 \cdot 0-0 \cdot 1)$ & $63 \cdot 0(55 \cdot 5-69 \cdot 8)$ & $1 \cdot 9(0 \cdot 3-10 \cdot 7)$ & $68 \cdot 5(52 \cdot 2-81 \cdot 2)$ & $0 \cdot 3(0 \cdot 1-1 \cdot 7)$ & $63 \cdot 9(56 \cdot 8-70 \cdot 4)$ \\
\hline Yes & $1 \cdot 1 * * *(0 \cdot 4-3 \cdot 2)$ & $59 \cdot 0(51 \cdot 9-65 \cdot 7)$ & $1 \cdot 1(0 \cdot 2-5 \cdot 9)$ & $55 \cdot 8(42 \cdot 8-68 \cdot 1)$ & $1 \cdot 1(0 \cdot 5-2 \cdot 7)$ & $58 \cdot 4(52 \cdot 0-64 \cdot 5)$ \\
\hline \multicolumn{7}{|c|}{ Sexual risk-taking practices (previous 12 months) } \\
\hline No & $0 \cdot 8(0 \cdot 2-3 \cdot 8)$ & $56 \cdot 5 *(50 \cdot 1-62 \cdot 7)$ & $2 \cdot 5(0 \cdot 9-6 \cdot 8)$ & $60 \cdot 7(47 \cdot 3-72 \cdot 6)$ & $1 \cdot 3(0 \cdot 5-3 \cdot 0)$ & $57 \cdot 6(51 \cdot 4-63 \cdot 6)$ \\
\hline Yes & $0 \cdot 6(0 \cdot 2-1 \cdot 7)$ & $66 \cdot 0 *(59 \cdot 6-71 \cdot 9)$ & $4 \cdot 7(1 \cdot 7-12 \cdot 1)$ & $52 \cdot 3(40 \cdot 6-63 \cdot 7)$ & $1 \cdot 5(0 \cdot 7-3 \cdot 4)$ & $62 \cdot 8(57 \cdot 3-68 \cdot 0)$ \\
\hline \multicolumn{7}{|c|}{ Self-reported HBV vaccination history } \\
\hline No & $0 \cdot 7(0 \cdot 2-2 \cdot 7)$ & - & $7 \cdot 4 *(2 \cdot 7-18 \cdot 6)$ & - & $2 \cdot 5(1 \cdot 0-5 \cdot 9)$ & - \\
\hline Yes & $0 \cdot 8(0 \cdot 3-2 \cdot 3)$ & - & $0 \cdot 6^{*}(0 \cdot 1-3 \cdot 4)$ & - & $0 \cdot 8(0 \cdot 3-1 \cdot 8)$ & - \\
\hline
\end{tabular}

PWUD, People who use drugs; CI, confidence interval; n.c., not concerned.

+ Vaccination history was estimated after exclusion of PWUD who were unaware of their vaccination status $(n=188)$ and non-respondents $(n=7)$.

$* P<0.05, * * P<0.01$, *** $P<0.001$. 
Table 4. Independent risk factors for HBs Ag positivity, ANRS-Coquelicot Survey 2011-2013 (multivariate

Poisson regression, $n=1575$ )

\begin{tabular}{lccc}
\hline \hline & PR & $(95 \%$ CI $)$ & $P$ \\
\hline $\begin{array}{l}\text { Endemic zone } \\
\quad \text { Low }\end{array}$ & Ref. & & \\
$\quad$ Moderate/high & $4 \cdot 81$ & $(1 \cdot 37-16 \cdot 84)$ & $0 \cdot 014$ \\
$\begin{array}{l}\text { Type of housing } \\
\quad \text { Stable }\end{array}$ & Ref. & & \\
$\quad \begin{array}{l}\text { Precarious } \\
\text { Syringe sharing (lifetime) }\end{array}$ & $3 \cdot 55$ & $(1-12 \cdot 92)$ & $0 \cdot 054$ \\
$\quad$ No & Ref. & & \\
$\quad$ Yes & $0 \cdot 84$ & $(0 \cdot 30-2 \cdot 36)$ & $0 \cdot 735$ \\
Sexual risk-taking practices (in the previous 12 & \\
months) & & & \\
$\quad$ No & Ref. & & \\
$\quad$ Yes & $1 \cdot 22$ & $(0 \cdot 34-4 \cdot 29)$ & $0 \cdot 759$ \\
\hline \hline
\end{tabular}

PR, Prevalence ratio; CI, confidence interval.

Conversely, overall, self-declared HBV vaccination history, estimated at $60 \cdot 9 \%(95 \%$ CI $56 \cdot 2-65 \cdot 4)$, decreased with the HBV endemicity level of participants' country of birth from $62.6 \%$ (95\% CI $57 \cdot 6-$ 67.4) for PWUD born in low endemic zones, to $47 \cdot 4 \%(95 \%$ CI $29 \cdot 9-65 \cdot 5)$ for those born in high endemic zones. To take into account these various epidemiological situations, and to identify the role of drug use in HBV infection, analyses were stratified for the endemicity level in the country of birth.

For PWUD born in low endemic countries, HBsAg seroprevalence was associated in univariate analysis with crack cocaine use in the previous month and with syringe sharing during one's lifetime. Sharing injection paraphernalia has already been shown to be one of the most important risk factors for HBV transmission [17]. No PWUD aged $<35$ years was infected with $\mathrm{HBV}$ whereas $1.0 \%$ of those aged $\geqslant 35$ years were infected (not significant). This is perhaps because the latter were less likely to have benefited from harm reduction measures [10]. HBsAg prevalence was also higher in those with an educational level below or equivalent to primary school and in those living in precarious housing conditions. Our results suggest that drug use and sharing drug injection paraphernalia are possible risk factors, among others, for HBsAg carriage. HBV infection seems to be multifactorial for PWUD born in low endemic zones. Due to insufficient numbers, multivariate analyses could not be performed on this population $(n=7)$. Accordingly, these results need to be confirmed in other larger studies.
Table 5. Independent factors associated with self-reported $H B V$ vaccination history ${ }^{\dagger}$, ANRS-Coquelicot Survey 2011-2013 (multivariate Poisson regression, $n=1362$ )

\begin{tabular}{|c|c|c|c|}
\hline & PR & $(95 \% \mathrm{CI})$ & $P$ \\
\hline \multicolumn{4}{|l|}{ Russian speaking } \\
\hline No & Ref. & & \\
\hline Yes & $0 \cdot 73$ & $(0 \cdot 54-0 \cdot 98)$ & $0 \cdot 039$ \\
\hline \multicolumn{4}{|l|}{ Age } \\
\hline$<35$ years & Ref. & & \\
\hline$\geqslant 35$ years & $0 \cdot 88$ & $(0 \cdot 77-0 \cdot 99)$ & $0 \cdot 046$ \\
\hline \multicolumn{4}{|l|}{ Living in a couple } \\
\hline No & Ref. & & \\
\hline Yes & $1 \cdot 06$ & $(0 \cdot 96-1 \cdot 17)$ & $0 \cdot 229$ \\
\hline \multicolumn{4}{|l|}{ Educational level } \\
\hline$>$ High school certificate & Ref. & & \\
\hline$<$ High school certificate & $1 \cdot 02$ & $(0 \cdot 89-1 \cdot 16)$ & $0 \cdot 762$ \\
\hline Primary school & $0 \cdot 63$ & $(0 \cdot 40-1)$ & $0 \cdot 052$ \\
\hline \multicolumn{4}{|l|}{ Syringe sharing (lifetime) } \\
\hline No & Ref. & & \\
\hline Yes & $0 \cdot 97$ & $(0 \cdot 86-1 \cdot 08)$ & $0 \cdot 545$ \\
\hline \multicolumn{4}{|c|}{ Self-perceived state of health } \\
\hline Poor & Ref. & & \\
\hline Good & $1 \cdot 19$ & $(1 \cdot 02-1 \cdot 40)$ & $0 \cdot 030$ \\
\hline \multicolumn{4}{|l|}{ HIV status } \\
\hline HIV Ab negative & Ref. & & \\
\hline HIV Ab positive & $0 \cdot 83$ & $(0 \cdot 64-1 \cdot 06)$ & $0 \cdot 135$ \\
\hline \multicolumn{4}{|l|}{$\mathrm{HCV}$ status } \\
\hline HCV Ab negative & Ref. & & \\
\hline $\mathrm{HCV} \mathrm{Ab} \mathrm{positive}$ & $0 \cdot 96$ & $(0 \cdot 84-1 \cdot 10)$ & $0 \cdot 556$ \\
\hline
\end{tabular}

PR, Prevalence ratio; CI, confidence interval.

$\dagger$ Vaccination history was estimated after exclusion of people who use drugs who were unaware of their vaccination status $(n=188)$ and non-respondents $(n=7)$

With respect to PWUD born in moderate and high $\mathrm{HBV}$-endemic countries, HBsAg prevalence was significantly higher in $\mathrm{HCV}$-seronegative PWUD and in those who had never injected. This suggests that drug injection is a minor additional risk factor for HBV infection for PWUD born in moderate or high HBV-endemic countries [5]. Indeed, in some African and Asian countries, in the absence of HBsAg antenatal screening and HBV immunization of newborns from infected mothers, the risk of perinatal transmission is high, as is the chronicity rate [3]. Consequently, being born in moderate or high endemic zones was the main factor associated with HBV chronic infection (PR 4.8) in multivariate analysis. This result is due to the higher risk of HBV infection and chronicity progression in moderate and high endemic countries compared to countries with low HBV prevalence. Results might have been different 
if we had studied the presence of anti-HBc antibodies, a marker of exposure to HBV, independent of the progression to chronicity.

In multivariate analysis, living in precarious housing conditions was also associated with HBsAg carriage. Unstable housing has already been shown to be a factor for increased injection risk behaviour, including injecting in public and receptive sharing of injecting paraphernalia [18] and, consequently, for increased hepatitis prevalence [10]. It may also be a confounding factor for sexual risk-taking. As the main initial objective of the ANRS-Coquelicot survey was to estimate $\mathrm{HCV}$ seroprevalence and associated factors, questions about sexual practices were few, and limited to the previous 12 months. The association between low socioeconomic status and HBV prevalence has also been previously described in the French general population [4].

Almost $60 \%$ of PWUD declared they had been vaccinated against $\mathrm{HBV}$, irrespective of the number of doses. This estimate is close to another calculated in 2010 in the subgroup of the French general population who declared drug injection at least once during their lifetime (52\%) [19]. In the UK in 2013, 72\% of PWID reported they were vaccinated against HBV [16]. Our estimate of self-reported vaccination history should be interpreted with caution as it probably overestimates HBV vaccine coverage. In a study conducted in PWID in the French region of Alsace in 2008-2009, vaccine coverage, based on HBs antibody serology, was estimated at only $28 \%$ [20]. Furthermore, in an Australian study published in 2010, although $31 \%$ of PWID declared they had been told by a health professional that they were fully vaccinated against HBV, only $52 \%$ of them had serological evidence of vaccination immunity [21]. This difference can be partly attributed to a lower immune response to hepatitis B vaccination in PWUD than in the general population [22], and partly due to both a low level of knowledge about hepatitis B [19] and a lack of awareness or poor understanding of their HBV status [23]. In the Australian study, only $61 \%$ of PWUD with serological evidence of vaccination knew that they were immunized [21]. In the ANRS-Coquelicot survey, fingerprick blood sampling on DBS was chosen to optimize PWUD participation in the study. However, this method prevented us from being able to detect $\mathrm{HBsAb}$ and $\mathrm{HBcAb}$ with accuracy and therefore to estimate the proportion of PWUD with serological evidence of HBV immunization, or the proportion of susceptible individuals who needed to be vaccinated. Despite these limitations, the associations highlighted in our study are consistent with the literature. In addition, HBsAg seroprevalence in PWUD who declared they were vaccinated was three times lower than in those who declared they had not been vaccinated, especially for people born in moderate or high HBV-endemic zones. Of participants born in low HBV-endemic countries, mainly in Metropolitan France, a self-reported HBV vaccination history was significantly more frequent in those aged $<35$ years. This generation may have benefited from the mass vaccination campaign between 1995 and 1998. In multivariate analysis, selfreported HBV vaccination history was associated with a good self-perceived state of health. Conversely, it was less frequently reported by those with only primary school education, irrespective of the endemicity level of their country of birth. Low coverage for HBV vaccination in PWUD has been previously shown to be partly attributable to economic and social barriers, including education level [24]. In our study, the less frequent self-reported vaccination history in those with only primary education may also be due to underreported vaccination arising from less knowledge about hepatitis in PWUD. Indeed, it has already been demonstrated in the general population that those with a lower educational level have less knowledge about hepatitis [19]. However, the higher HBsAg prevalence among PWUD with low educational qualifications suggests there is a real difference in HBV vaccine coverage according to education level. Lower vaccine coverage in Russian-speaking PWUD might be due to vaccine policies in Russian-speaking countries which are very different from those in France.

The ANRS-Coquelicot survey suggested that HBV vaccine coverage in PWUD may be less than optimal. Many studies have documented the concomitant decrease of hepatitis B infection with increased HBV immunization rates $[25,26]$. PWUD vaccine coverage may be improved in two ways. First, efforts to increase immunization in the French general population must continue and be intensified, especially in adolescents, a sub-population where only $50 \%$ are currently immunized [27]. Second, HBV immunization targeted at PWUD has to be reinforced, and as soon as possible after the start of illicit drug use. To achieve this goal, many actions have already demonstrated their efficiency: providing free vaccination in specialized centres attended by PWUD, improving training and increased knowledge for healthcare workers 
regarding $\mathrm{HBV}$ and its prevention [20], implementing an accelerated HBV vaccination schedule [28, 29], offering financial incentives to PWUD to enhance their adherence to the HBV vaccine schedule [30, 31], increasing PWUD knowledge about HBV, and providing greater access to hepatitis screening [24].

Some study limitations have to be discussed. Due to the low number of HBsAg-positive PWUD, the analyses - in particular the multivariate analysis - were limited and suffered from a lack of power. Since our study recruited PWUD in specialized centres, it did not provide information on 'hidden' PWUD who did not attend these structures. Finally, the study was implemented in geographical areas which were not randomly chosen. However, these zones were selected to reflect the diversity of drug use in France. Moreover, PWUD were selected at random.

In conclusion, the HBsAg prevalence estimated in PWUD in the ANRS-Coquelicot survey was moderate, but not negligible, especially in those born in high HBV-endemic countries. Our results suggest that drug use may be a moderate contributing factor to HBsAg carriage in PWUD, especially in those from high $\mathrm{HBV}$ prevalence countries.

\section{APPENDIX. ANRS Coquelicot Survey Group}

F. Barin, T. Benoit, S. Brunet, S. Chevaliez, M. Jauffret-Roustide, L. Léon, Y. Le Strat, M. Molinier, J.-M. Pawlotsky, J. Pillonel, C. Semaille, C. Sommen, A. Soulier, D. Thierry, L. Weill-Barillet.

\section{ACKNOWLEDGEMENTS}

The authors acknowledge all the PWUD who agreed to participate in the study and all the professionals from the centres that made the ANRS-Coquelicot survey possible.

We thank C. Aranda, G. Guibert, L. Oudaya, B. Schuch and the ADR 11 Inserm team for the preparation and administrative follow-up of the survey.

We also extend our thanks to all the interviewers who contributed to data collection: Bordeaux: Françoise Bordes, Patrick Chambreau, Eugène Martinheira, Emanuel Rappeneau. Lille: Josué Bogarim, Véronique Botte, Nadia Goune, Samia Jbara, Anne Luyckx. Marseille: Muriel Garnier, Christophe Goby, Pierre Grimal, Sandrine Huguet, Gisèle Lafforgue, Henri Sigaud. Paris and Ile-de-France: Jean-Marc Benhaim, Gérard Brodsky,
Agnès Chetaille, Anne Darras, Patrice Dauvergne, Véronique David Dehal, Christian Diaz, Vincent Ferre, Stéphane Golenischev, Emmanuel Guillais, Sylvie Guillien, Patricia Jaccaz, Mireille Le Breton, Virginie Le Gall, Michelle Roussier, Aude Saintier, Michelle Tilloy, Patricia Vergnes. Strasbourg: Josefa Keck, Nicole Olivier Martin.

We also thank Marion Fleury for the first version of the translation from French to English and Jude Sweeney for the final editing.

This study received financial support from: ANRS (French National Agency for AIDS and Hepatitis Research), French Public Health Agency (Santé publique France), French Ministry of Heath - HIV Division.

\section{DECLARATION OF INTEREST}

P. Sogni: Boards, workshops and meeting invitations: Gilead, BMS, AbbVie, Janssen, MSD, Mayoly-Spindler.

\section{REFERENCES}

1. Schweitzer A, et al. Estimations of worldwide prevalence of chronic hepatitis B virus infection: a systematic review of data published between 1965 and 2013. Lancet 2015; 386: 1546-1555.

2. GBD 2013 Mortality and Causes of Death Collaborators. Global, regional, and national age-sex specific all-cause and cause-specific mortality for 240 causes of death, 1990-2013: a systematic analysis for the Global Burden of Disease Study 2013. Lancet 2015; 385: 117-171.

3. Locarnini S, et al. Strategies to control hepatitis B: Public policy, epidemiology, vaccine and drugs. Journal of Hepatology 2015; 62: S76-86.

4. Meffre C, et al. Prevalence of hepatitis B and hepatitis C virus infections in France in 2004: social factors are important predictors after adjusting for known risk factors. Journal of Medical Virology 2010; 82: 546-555.

5. World Health Organization. Hepatitis B. Fact sheet no. 204 (http://www.who.int/mediacentre/factsheets/fs204/ en/). Accessed 19 May 2016.

6. Denis F, Levy-Bruhl D. Mass vaccination against hepatitis B: the French example. Current Topics of Microbiology and Immunology 2006; 304: 115-129.

7. World Health Organization. 63rd World Health Assembly: Resolution A63/15: Viral Hepatitis (http:// apps.who.int/gb/ebwha/pdf_files/WHA63/A63_15-en. pdf). Accessed 23 May 2016.

8. Mathers BM, et al. Global epidemiology of injecting drug use and HIV among people who inject drugs: a systematic review. Lancet 2008; 372: 1733-1745.

9. Weill-Barillet L, et al. Hepatitis C virus and HIV seroprevalences, sociodemographic characteristics, behaviors and access to syringes among drug users, a comparison 
of geographical areas in France, ANRS-Coquelicot 2011 survey. Revue d'Epidemiologie et de SantéPublique 2016; 64: 301-312.

10. Jauffret-Roustide $\mathbf{M}$, et al. A national cross-sectional study among drug-users in France: epidemiology of HCV and highlight on practical and statistical aspects of the design. BMC Infectious Diseases 2009; 9: 113.

11. Mohamed S, et al. Dried blood spot sampling for hepatitis B virus serology and molecular testing. PLoS ONE 2013; 8: e61077.

12. Njai HF, et al. Validation of rapid point-of-care (POC) tests for detection of hepatitis B surface antigen in field and laboratory settings in the Gambia, Western Africa. Journal of Clinical Microbiology 2015; 53: 1156-1163.

13. Chan HL, et al. Hepatitis B surface antigen quantification: why and how to use it in 2011 - a core group report. Journal of Hepatology 2011; 55: 1121-1131.

14. European Monitoring Center for Drugs and Drug Addiction. HBsAg prevalence. European Data 2015 (http://www.emcdda.europa.eu/data/stats2015). Accessed 4 June 2016.

15. Lugoboni F, et al. Let It 'B'? The role of hepatitis B universal vaccination among italian problematic drug users. International Journal of Environmental Research and Public Health 2015; 12: 3979-3992.

16. Public Health England. Data tables of the Unlinked Anonymous Monitoring Survey of HIV and Hepatitis in People Who Inject Drugs 2014 (https://www.gov.uk/ government/uploads/system/uploads/attachment_data/ file/326897/UAM_Survey_of_PWID_data_tables_2014. pdf). Accessed 28 May 2016.

17. Bialek SR, et al. Risk factors for hepatitis B in an outbreak of hepatitis B and D among injection drug users. Journal of Urban Health 2005; 82: 468-478.

18. Des Jarlais DC, Braine N, Friedmann P. Unstable housing as a factor for increased injection risk behavior at US syringe exchange programs. AIDS and Behavior 2007; 11: 78-84.

19. Brouard C, et al. Hepatitis B knowledge, perceptions and practices in the French general population: the room for improvement. BMC Public Health 2013; 13: 576.

20. Di Nino F, et al. Evolution of hepatitis B vaccination coverage among psychoactive substances users followed by the Network of Medical Microstructures in Alsace
(France), 2009-2012. Bulletin Epidemiologique Hebdomadaire 2014; 11: 192-200.

21. Day CA, et al. Hepatitis B virus among injecting drug users in Sydney, Australia: prevalence, vaccination and knowledge of status. Drug and Alcohol Dependence 2010; 108: 134-137.

22. Kamath GR, Shah DP, Hwang LY. Immune response to hepatitis B vaccination in drug using populations: a systematic review and meta-regression analysis. Vaccine 2014; 32: 2265-2274.

23. Topp L, et al. Poor criterion validity of self-reported hepatitis $\mathrm{B}$ infection and vaccination status among injecting drug users: a review. Drug and Alcohol Revue 2009; 28: 669-675.

24. Quaglio G, et al. Hepatitis vaccination among drug users. Vaccine 2006; 24: 2702-2709.

25. Judd A, et al. Twenty years of selective hepatitis B vaccination: is hepatitis B declining among injecting drug users in England and Wales? Journal of Viral Hepatitis 2007; 14: 584-591.

26. Mossner BK, et al. Decline in hepatitis B infection observed after 11 years of regional vaccination among Danish drug users. Journal of Medical Virology 2010; 82: $1635-1639$.

27. Guthmann JP, et al. Hepatitis B vaccination coverage among children in France in 2014: substantial progress in infants, stagnation in adolescents. Bulletin Epidemiologique Hebdomadaire 2015; 26-27: 499-504.

28. Bowman S, et al. Factors associated with hepatitis B vaccine series completion in a randomized trial for injection drug users reached through syringe exchange programs in three US cities. BMC Public Health 2014; 14: 820 .

29. Shah DP, et al. Long-term effectiveness of accelerated hepatitis B vaccination schedule in drug users. American Journal of Public Health 2015; 105: e36-43.

30. Weaver T, et al. Use of contingency management incentives to improve completion of hepatitis B vaccination in people undergoing treatment for heroin dependence: a cluster randomised trial. Lancet 2014; 384: 153-163.

31. Topp $\mathbf{L}$, et al. A randomised controlled trial of financial incentives to increase hepatitis B vaccination completion among people who inject drugs in Australia. Preventive Medicine 2013; 57: 297-303. 